\title{
Enhancing workers safety in worksites through augmented GNSS sensors
}

Mauro D’Arco, Alfredo Renga, Andrea Ceccarelli, Francesco Brancati, Andrea Bondavalli

\begin{abstract}
Human-assistive wearable technologies can monitor the position of a worker in a critical worksite and improve his safety by raising warnings when he is in a dangerous zone. In railway worksites, it is observed that GNSS localization is not applicable due to the relevant localization errors, which are only partially attributable to the specific characteristics of the worksite. Besides, alternative solutions that rely on the support of anchors have the drawback of lengthening the setup and dismantle time of the worksite. To provide safe localization of railway trackside workers, a system that exploits augmented-GNSS wearable sensors is proposed. Each sensor includes a GPS receiver capable of sharing its coordinates with the other sensors, and is further complemented with a low-cost multi-way distance meter to measure its distance from the other sensors. The main attention is paid to the data fusion approach adopted to improve the accuracy of the position estimate by combining the relative distance measurements to the GPS coordinates.
\end{abstract}

Keywords - GNSS, localization, wearables, safety, accuracy, positioning, railway trackside workers, 


\section{INTRODUCTION}

Self-localization capability is a highly desirable characteristic of mobile electronic devices. It enables a myriad of applications such as inventory management, intrusion detection, road traffic monitoring, health monitoring, reconnaissance, surveillance [1]. Nonetheless, self-localization is also an attractive mean to guarantee safety of people or moving assets, when they operate in critical areas in which there is a significant risk of being struck by objects or vehicles [2].

The most straightforward approach to achieve self-localization relies on the Global Navigation Satellite System (GNSS) i.e., satellite-based localization as the Global Positioning System (GPS [3]). However, in several environments, satellite-based localization can result challenging, because of specific characteristics of the environment, or difficulties of assuring tight accuracy requirements: GNSS sensors localization errors can in fact be even greater than a few meters [4].

An alternative solution is adopted in air traffic surveillance applications. Specifically, a dedicated technology, known as Automatic Dependent Surveillance-Broadcast (ADS-B) [5], is preferred to GNSS or radar based systems to accurately track airplanes in flight and on the ground. Airport surface surveillance takes advantage of ADS-B to assure safety of workers by providing situational awareness about the airport surface dynamics during cargo and shuttle operations. Unfortunately, ADS-B solutions are less flexible that GNSS ones and cannot be easily reproduced outside the airport ramp; typically, they cannot be enabled in worksites either for budget limitations or technical constraints.

More and more often, GNSS based solutions are combined with other systems in order to obtain increased accuracy and allow workers position tracking in critical worksites. As an example, in railway worksites effective tracking has been managed through a GPS system that combines GPS data with information from electronic fences placed within the worksite area [6]. Specifically, the fences data are collected from the communication layer, and fused with the GPS data in the localization component [7]. This allows determining with high accuracy if the worker is in a safe (green) or unsafe (red) zone. However, the set-up and dismantle time of fences is a significant limitation: such a solution is impractical when the worksite is established only for a short time or when it is frequently moved along the railway [8]-[10].

In this paper, a GNSS-augmentation approach is proposed to improve self-localization of workers, with the specific objective of detecting if they are located in a dangerous area. The focus of the work is the railway domain, although other domains could be targeted as well. In the railway worksite, ground infrastructure may not be available due to the characteristic of the surrounding environment. Cost constraints usually force to disregard expensive equipment, as well as anchor-based systems, since they affect the set-up and dismantle time of the worksite. The main target is therefore the definition of a solution for human-assistive wearables, that can be realized by means of devices with low cost, reduced weight, and limited power consumption such that daily operability can be assured.

The considered system is composed of wearable GNSS sensors that include multi-way distance sensors and allow data sharing. Each wearable sensor is assigned to a worker and performs fusion of the GNSS receivers data and its own multi-way distance sensor data to provide an estimate of the positions of the workers. The proposed GNSS augmentation approach is analytically presented in Section II. The achievable performance is evaluated and compared to that offered by GNSS in Section III, where it is shown that thanks to the proposed solution it is possible to identify workers in safe and unsafe zones, reducing the number of false and missed alarms with respect to systems exclusively based on the GNSS technology. Also, compliance with standard regulations and applicability to the railway domain are discussed in Section IV. Finally, concluding remarks are given in Section IV. 


\section{PROPOSED GNSS AUGMENTATION APPROACH}

The proposed approach exploits a data fusion algorithm to combine measured data and estimate the positions of the workers . Each worker is equipped with a GNSS receiver and a distance sensor, included into a single device. For the sake of clarity, a simplified version of the data fusion algorithm is first described under the assumption of fully connected network i.e., each device can measure its distance from all the others. Later on, the assumption is removed and the proposed algorithm straightforwardly improved.

The proposed algorithm computes the absolute positions of all the workers by combining the data measured by all the devices. In detail, the vector of the measurement data $\mathbf{y}$, includes the data from the GNSS receivers and the distance sensors. For a team of $N$ workers, each one equipped with a correspondent device, the number of elements in the vector $\mathbf{y}$ is $3 N+N(N-1)$, since there are $3 N$ coordinates from the GNSS receivers, and $N-1$ distance values from each distance sensor.

The vector $\mathbf{y}$ is related through an observation model $\mathbf{h}$ to the vector of the positions of the workers $\mathbf{x}$, which includes $3 N$ elements, by:

$$
\mathbf{y}=\mathbf{h} \mathbf{x}+\mathbf{v}
$$

where vector $\mathbf{v}$ represents additive Gaussian noise. The observation model $\mathbf{h}$ is non-linear since its coefficients depend on the positions of the devices. If the initial positions $\mathbf{x}_{0}$ of the workers are roughly known (to this end the data offered by the GNSS receivers can be considered without any data fusion improvement), the knowledge of their actual positions can be improved and tracked at the successive time epochs by solving linear problems, which include the fusion of the data coming from GNSS receivers and the distance sensors. In fact, equation (1) can be linearized in the neighboring of the initial state $\mathbf{x}_{0}$ according to:

$$
\mathbf{y}_{1}=\mathbf{y}_{0}+\Delta \mathbf{y}_{1}=\mathbf{h}\left(\mathbf{x}_{0}\right) \mathbf{x}_{\mathbf{0}}+\mathbf{J}_{\mathbf{h}}\left(\mathbf{x}_{0}\right) \Delta \mathbf{x}_{1}
$$

where the current measurements $\mathbf{y}_{\mathbf{1}}$ are represented in terms of expected values $\mathbf{y}_{0}$, evaluated by means of the observation model as $\mathbf{y}_{0}=\mathbf{h}\left(\mathbf{x}_{0}\right) \mathbf{x}_{0}$, plus a difference, $\Delta \mathbf{y}_{1}$. This difference is linearly related to a correction term $\Delta \mathbf{x}_{1}$ for the positions of the workers through the Jacobian of the observation model, evaluated in the initial state $\mathbf{J}_{\mathbf{h}}\left(\mathbf{x}_{0}\right)$. The positions of the workers will be evaluated at the generic time epoch $k$ as $\mathbf{x}_{\mathrm{k}}=\mathbf{x}_{\mathrm{k}-1}+\Delta \mathbf{x}_{\mathrm{k}}$, where the correction term $\Delta \mathbf{x}_{\mathrm{k}}$ is gained by inverting the linear problem:

$$
\Delta \mathbf{y}_{k}=\mathbf{J}_{\mathbf{h}}\left(\mathbf{x}_{k-1}\right) \Delta \mathbf{x}_{k}
$$

In particular, $\Delta \mathbf{x}_{\mathrm{k}}$ is obtained using a best linear unbiased estimator, which is substantially a weighted least square estimator, designed to provide a maximum likelihood estimate. To this end, equation (3) is inverted using an auxiliary weighting matrix, known as measurement information matrix, which is the inverse of the covariance matrix $\mathbf{R}$ of the noise affecting the measured data. As shown in [11], the best linear unbiased estimate of $\Delta \mathbf{x}_{\mathrm{k}}$ is:

$$
\Delta \mathbf{x}_{k}=\left(\mathbf{J}_{\mathbf{h}}^{T} \mathbf{R} \mathbf{J}_{\mathbf{h}}\right)^{-1} \mathbf{J}_{\mathbf{h}}^{T} \mathbf{R}^{-1} \Delta \mathbf{y}_{k}
$$


where the superscript $T$ stands for transpose operator. In equation (4) all the measured variables are supposed to be independent from each other and affected by Gaussian noise of known variance; the Jacobian $\mathbf{J}_{\mathbf{h}}$ is evaluated considering the positions of the workers at the time epoch $k-1$.

It is worth noting that the time epoch grid characterizing the processing operations can be much finer than that characterizing the measurements updates. In this case, the system benefits of the additional iterations of the proposed algorithm that definitely improve the estimates. To reduce power consumption, the iterations can be repeated until a stopping criterion, based on the norm of $\Delta \mathbf{x}_{\mathrm{k}}$, is met; then the system is put in sleeping mode waiting for the update of the measurement data $\mathbf{y}_{\mathrm{k}+1}$, which will allow to compute $\Delta \mathbf{y}_{\mathrm{k}+1}$ and restart the processing. In more realistic scenarios, the observation model should be dynamically configured, by including or excluding some rows, when the distance sensors data or GPS coordinates are not available. In fact, the distance sensors have a limited range of operation, and can even fail their task if the target is within the operation range but not in line of sight. Consequently at the generic time epoch only a subset counting $M$ out of $N-1$ distances can be available from each distance sensor. This implies that the wearable devices use differently-configured observation models.

\section{PERFORMANCE ANALYSIS}

Manufacturers assess their GNSS receivers performance distinguishing between horizontal and vertical directions. The latter performance is typically much worse than the former; fortunately, accurate knowledge of the vertical position is not crucial in the great majority of cases.

The parameters commonly used to express the performance in the horizontal plane are: circular error probable 50\% (CEP50), radius 95\% (R95), root mean square (RMS), and two times the distance of RMS (2DRMS) [12]. These parameters represent the radius of a circular region in the horizontal plane, where the positions gained by the receiver during on-field experiments are found with a given probability, that is 50\% for CEP50, 95\% for R95, 68\% for RMS and 95\% for 2DRMS. The performance in the vertical direction is expressed as a factor times that of the horizontal plane [13], and has to be intended as the length of a linear segment where the vertical position is found with the same confidence of the horizontal parameter.

In detail, experimental assessment of GNSS receivers performance requires gathering a 24-hour, or longer, data set of positions of static GNSS receivers, in order to log the diurnal environmental effects (e.g. ionosphere) and visibility conditions of GNSS satellites. The data set of positions is then compared either to certified surveyed reference markers or to the centroid of the same data set to estimate the performance parameters. .

It is worth noticing that these performance parameters cannot be regarded as absolute indicators of the positioning uncertainty, which is not uniquely related to the quality of the receiver. They are instead relative in nature, since they depend on both the test site and the period of the year, and lack of reproducibility: they can significantly vary due to ionosphere and troposphere influences, number and angular distribution of available GNSS satellites, , on-board satellite ephemeris and clock uncertainty, noise, signal multipath effects and so on. Nonetheless, they are accepted by customers as marks of the quality of different products, being useful to compare different receivers, especially if tested in the same experimental session [14].

Hereinafter, the performance analysis of the proposed approach is carried out by means of a simulation tool, that allows determining the positions of a set of GNSS sensors, and comparing the results to those gained using augmented GNSS sensors. Simulation results are given in terms of position errors and RMS values of the distance, in the horizontal plane, from detected to true positions. Also, false and missed alarm rates of both solutions are compared in order to highlight the advantages offered by the proposed one in the considered application. 


\section{A. Simulation tool}

A simulation tool has been developed to evaluate the performance of the proposed data fusion algorithm. The proposed tool can take into account different worksites and workers distributions within the worksite, and it is able to simulate both ranging measurements and GNSS observables. It includes and complements the Satellite Navigation Toolbox 3.0 for Matlab® developed by GPSoft, Inc [15], adopted to simulate the GNSS satellite constellations, the propagation environment (ionosphere, troposphere, multipath) and the receiver measurements (noise and bias).

Standard stand-alone single-frequency GNSS operation [16] is assumed, with receiver position evaluated as ordinary leastsquare solution of pseudorange measurements, after partial compensation of propagation delays, using broadcast ephemeris. Pseudorange measurements are also then fed to the algorithm presented in Section II to perform GNSS augmentation by ranging measurements. Ranging measurement errors are simulated assuming unbiased Gaussian distribution along the Line of Sight (LoS) between two receivers. Table I resumes and discusses the main assumptions of the developed simulation environment. It is important to note that ranging sensors are assumed to have a limited spherical maximum range, both for communication and for positioning, so, depending on the selected scenario, the number of available ranging measurements is in general different for each worker within the worksite.

\section{B. Simulated worksite description}

The simulated worksite is a rectangular area $1000 \mathrm{~m}$ x $200 \mathrm{~m}$ wide including a green zone and a red zone. The center of the area is located at a mid-latitude region in the northern hemisphere $\left(40.81^{\circ} \mathrm{N}\right.$ latitude, $14.14^{\circ} \mathrm{E}$ longitude) and represents the origin of the introduced local east-north-up (ENU) reference frame. The longest size of the site is along the west-east direction, the shortest is in the south-north one. The red zone is a stripe $1000 \mathrm{~m}$ long and $20 \mathrm{~m}$ wide supposed to include tracks and nearby areas. The worksite includes 13 randomly distributed workers. Four workers operate within the red zone, and two workers are placed less than 3 meters from the green zone. The remaining workers are in the green zone, but some of them are relatively close to the red one. Specifically, less than $3 \mathrm{~m}$ distance from the red zone is assumed for three workers operating in the green zone. Hence, the simulated scenario, illustrated in Figure 1, is expected to be prone to the generation of both false positives and false negatives when only GNSS is used.

TABLE I- AsSUMPTIONS AND Key NEGLECTED EFFECTS.

\begin{tabular}{|c|c|l|l|l|}
\hline Assumption & $\#$ & Details & $\begin{array}{l}\text { Simplification } \\
\text { Achieved }\end{array}$ & Key Neglected Effects \\
\hline & 1 & GNSS measures pseudoranges & & Second order effects \\
\cline { 2 - 5 } & 2 & $\begin{array}{l}\text { Different sensors have realistically correlated } \\
\text { errors }\end{array}$ & & Second order effects \\
\cline { 2 - 5 } model & 3 & $\begin{array}{l}\text { Unbiased Gaussian pseudorange noise } \\
\text { distribution }\end{array}$ & $\begin{array}{l}\text { Allows for linear } \\
\text { analysis }\end{array}$ & $\begin{array}{l}\text { Systematic errors are present in GNSS computed pseudoranges. } \\
\text { These are due to atmospheric propagation delays, SV and } \\
\text { receiver hardware effects, etc. Careful compensation of these } \\
\text { systematic effects can yield a Gaussian-like error distribution. }\end{array}$ \\
\hline $\begin{array}{c}\text { Ranging } \\
\text { error model }\end{array}$ & 4 & $\begin{array}{l}\text { Unbiased Gaussian error distribution in the } \\
\text { LoS range. }\end{array}$ & $\begin{array}{l}\text { Allownores systematic errors such as hardware effects. } \\
\text { analysis }\end{array}$ \\
\hline
\end{tabular}




\begin{tabular}{|c|c|c|c|c|}
\hline & 5 & Different sensors have uncorrelated errors & $\begin{array}{l}\text { Mathematical } \\
\text { Simplicity }\end{array}$ & $\begin{array}{l}\text { Correlation is introduced by signal propagation, multipath, etc. } \\
\text { Its magnitude depends on the specific ranging system assumed. }\end{array}$ \\
\hline & 6 & $\begin{array}{l}\text { Measurement accuracy does not depend on } \\
\text { distance }\end{array}$ & $\begin{array}{l}\text { Mathematical } \\
\text { Simplicity }\end{array}$ & $\begin{array}{l}\text { This assumption is questionable for ranging sensors based on } \\
\text { power level detection. Those based on Time-of-Arrival are less } \\
\text { sensitive to this effect. }\end{array}$ \\
\hline & 7 & $\begin{array}{l}\text { Sensors are assumed to have a limited spherical } \\
\text { maximum range (both for communication\& for } \\
\text { positioning) }\end{array}$ & $\begin{array}{l}\text { Mathematical } \\
\text { Simplicity }\end{array}$ & Depends on the scenario we will use \\
\hline $\begin{array}{c}\text { Devices } \\
\text { capabilities }\end{array}$ & 8 & $\begin{array}{l}\text { All devices have the same positioning sensors } \\
\text { (e.g. GNSS, Ranging) }\end{array}$ & $\begin{array}{l}\text { Mathematical } \\
\text { Simplicity }\end{array}$ & Depends on the chosen scenario. \\
\hline \multirow{3}{*}{$\begin{array}{l}\text { Correlation } \\
\text { In Time }\end{array}$} & 9 & Devices are stationary in ENU & $\begin{array}{l}\text { Allows for a single } \\
\text { epoch algorithm }\end{array}$ & $\begin{array}{l}\text { Applies to scenarios in which the devices position does not } \\
\text { change in time (Static devices), or does not change appreciably } \\
\text { between consecutive time-epoch compared to positioning errors } \\
\text { magnitude. }\end{array}$ \\
\hline & 10 & $\begin{array}{l}\text { All devices share updated position information } \\
\text { at each time epoch }\end{array}$ & $\begin{array}{l}\text { Allows for a single } \\
\text { epoch algorithm }\end{array}$ & $\begin{array}{l}\text { Applies to scenarios in which the positioning task is solved by } \\
\text { each device at a rate well below the information-sharing one. } \\
\text { GNSS-based applications usually perform positioning @ } 1 \mathrm{~Hz} \text {, } \\
\text { making this assumption realistic in most cases. }\end{array}$ \\
\hline & 11 & $\begin{array}{l}\text { Errors are white noises, i.e., no correlation in } \\
\text { time exists between consecutive measurements }\end{array}$ & $\begin{array}{l}\text { Allows for a single } \\
\text { epoch algorithm }\end{array}$ & $\begin{array}{l}\text { Depends on sensor model and positioning data rate. Usually, } \\
\text { GNSS measurements are considered uncorrelated up to } \mathrm{O}(1 \mathrm{~Hz}) \\
\text { output data rates. }\end{array}$ \\
\hline
\end{tabular}

\section{GNSS performance}

One-day long simulation is performed assuming workers at fixed location. In this way, GNSS performance can be evaluated in different conditions in terms of both geometric dilution of precision (GDOP) and signal propagation (ionospheric and tropospheric delays). Baseline GPS constellation (24 satellites) is simulated. Figure 2 shows the number of tracked GPS satellites and the relevant estimated GDOP for the receiver placed at the origin of coordinates. Satellite availability can be considered satisfactory, with tracked satellites ranging from 6 to 11. GDOP ranges from less than 2 to more than 3 for most of the simulated time span. However, about 1 hour time period with GDOP time larger than 7 also occurs. 

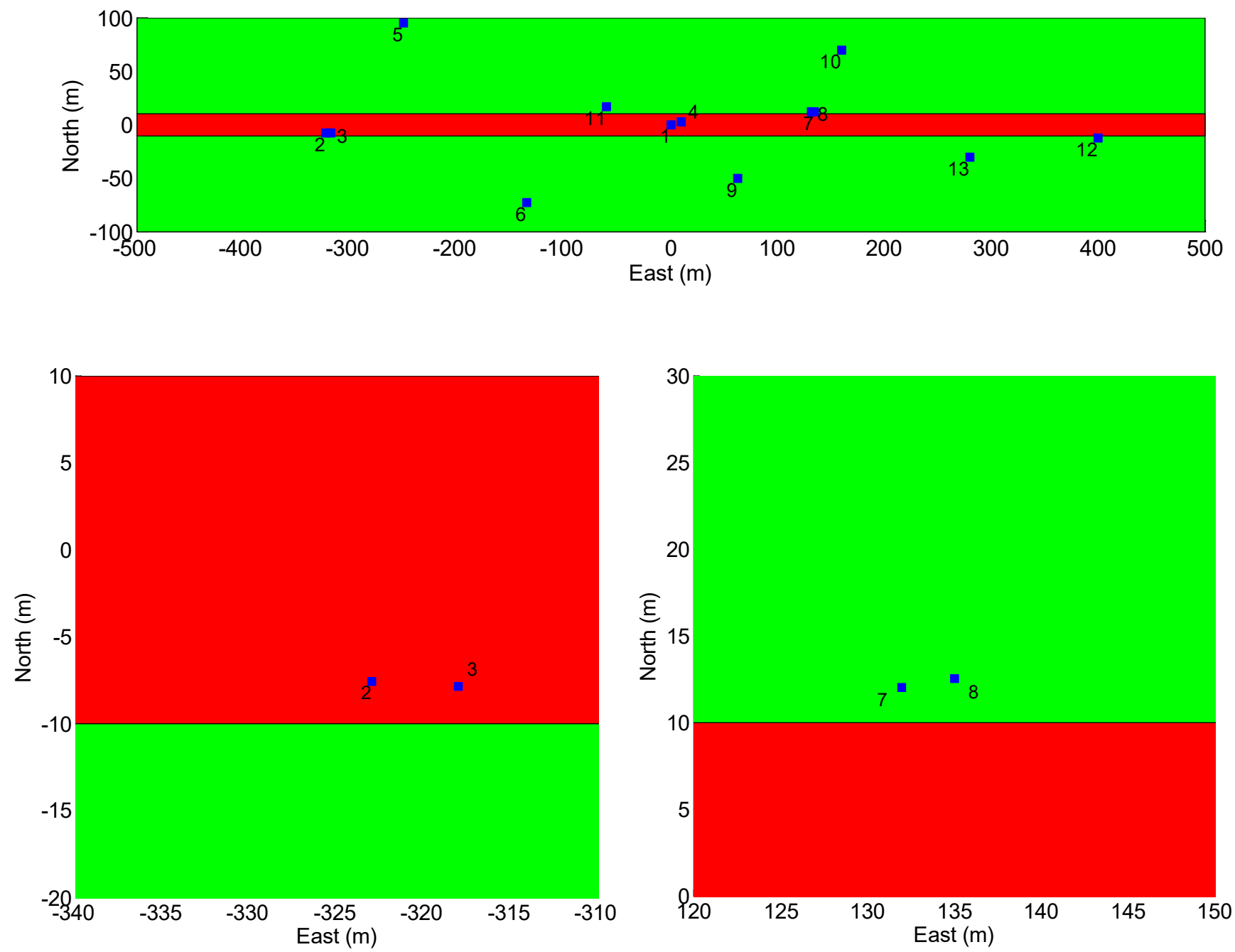

Figure 1 (Top) Simulated worksite highlighting green and red zone. Workers indicated by blue markers and relevant index number. (Bottom Left) Zoomed view of two workers operating in the red zone but close to the red-to-green zone boundary. (Bottom Right) Zoomed view of two workers operating in the green zone but close to the red-to-green zone boundary. 


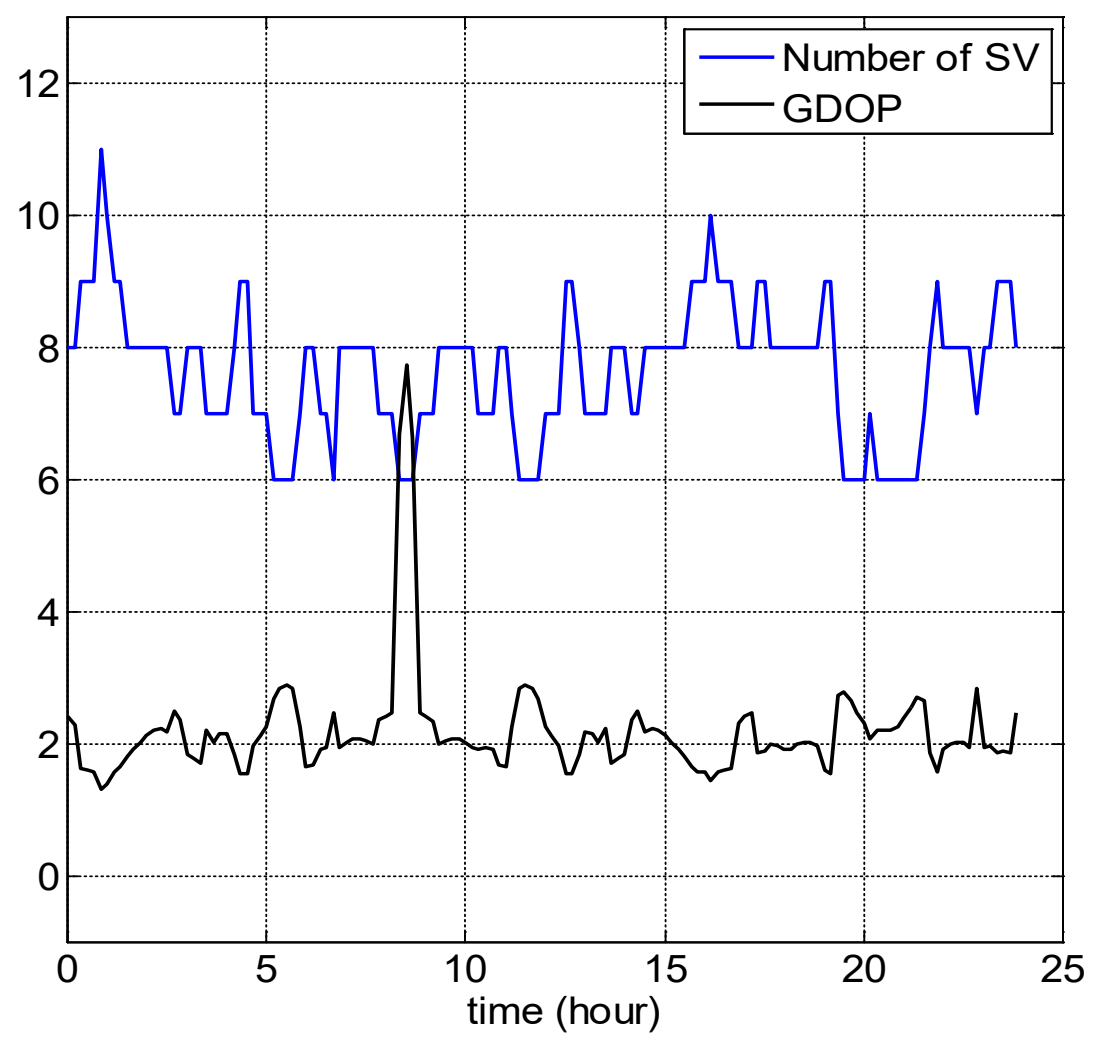

Figure 2 Number of tracked GNSS satellites (blue) and GDOP (green) in the simulated one-day long time period. Results refer to the receiver located at the origin of coordinates.

Figures 3-4 show positioning performance considering GNSS only and assuming $0.35 \mathrm{~m}$ pseudorange measurements noise. As expected, errors along the vertical components are more significant than those in the east-north plane. Up to $30 \mathrm{~m}$ vertical errors can be experienced owing to satellite geometry and limited accuracy of compensated measurement biases and propagation delays resulting from the standard single-frequency standalone positioning algorithm. Concerning the results of Figures 3-4, it is worth noting that most of the errors are space-correlated i.e., they are shared by different workers in the worksite. Differential errors generated by receiver noise and spatial separation between the receivers are smaller but still significant, ranging from one to several meters along each axis. The RMS value of the distance from the true to the estimated positions is about $2.7 \mathrm{~m}$ for all the simulated workers. 


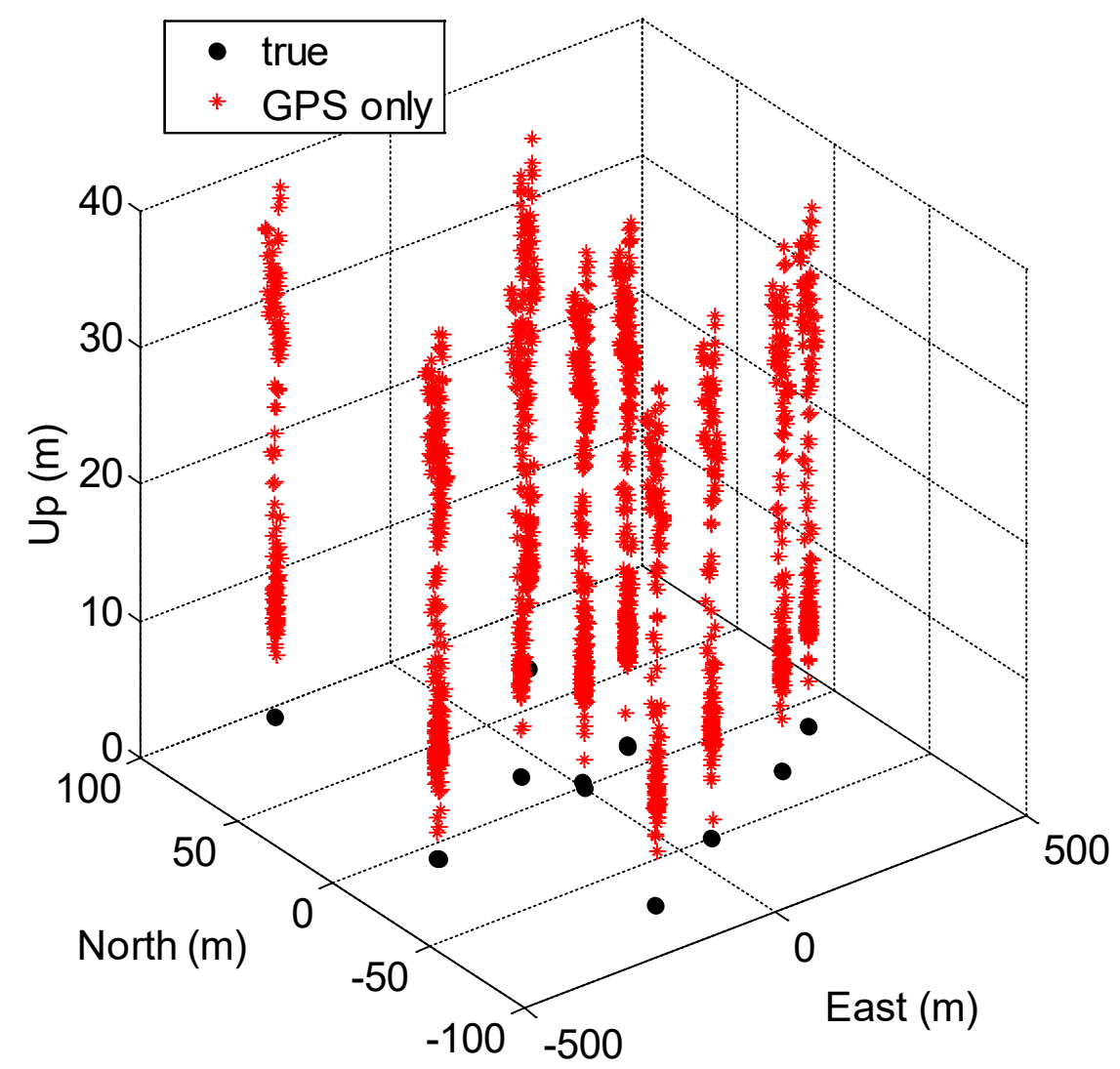

Figure 3. 3D GNSS error for each receiver in the worksite.

Figure 5 shows examples of GNSS-only positioning accuracy for some workers in close proximity to green-to-red zone boundaries. In the simulated scenario, 22 false positives are generated together with 15 false negative. Specifically, false positives are generated by workers with index number 7,8 , and 12 , respectively, whereas false negative are generated by workers with index 2 and 3.

\section{Augmented GNSS performance}

Fusion of GNSS and ranging measurements can be properly exploited to reduce both false positive rate and, more important, the rate of false negative.

Figure 6 shows, for each worker, the circle in the east-north plane including usable ranging devices assuming 250 m maximum range. It is clear that, depending on the selected distribution of workers in the worksite, the number of ranging measurements that each device can exploit is different.

In the considered case (Figure 7) of randomly distributed workers and $250 \mathrm{~m}$ maximum range, the number of available ranging measurements is 8 for receivers 9 and 11, but it drops to 1 for receiver 12 (which can only exchange range measurements with worker 13). 

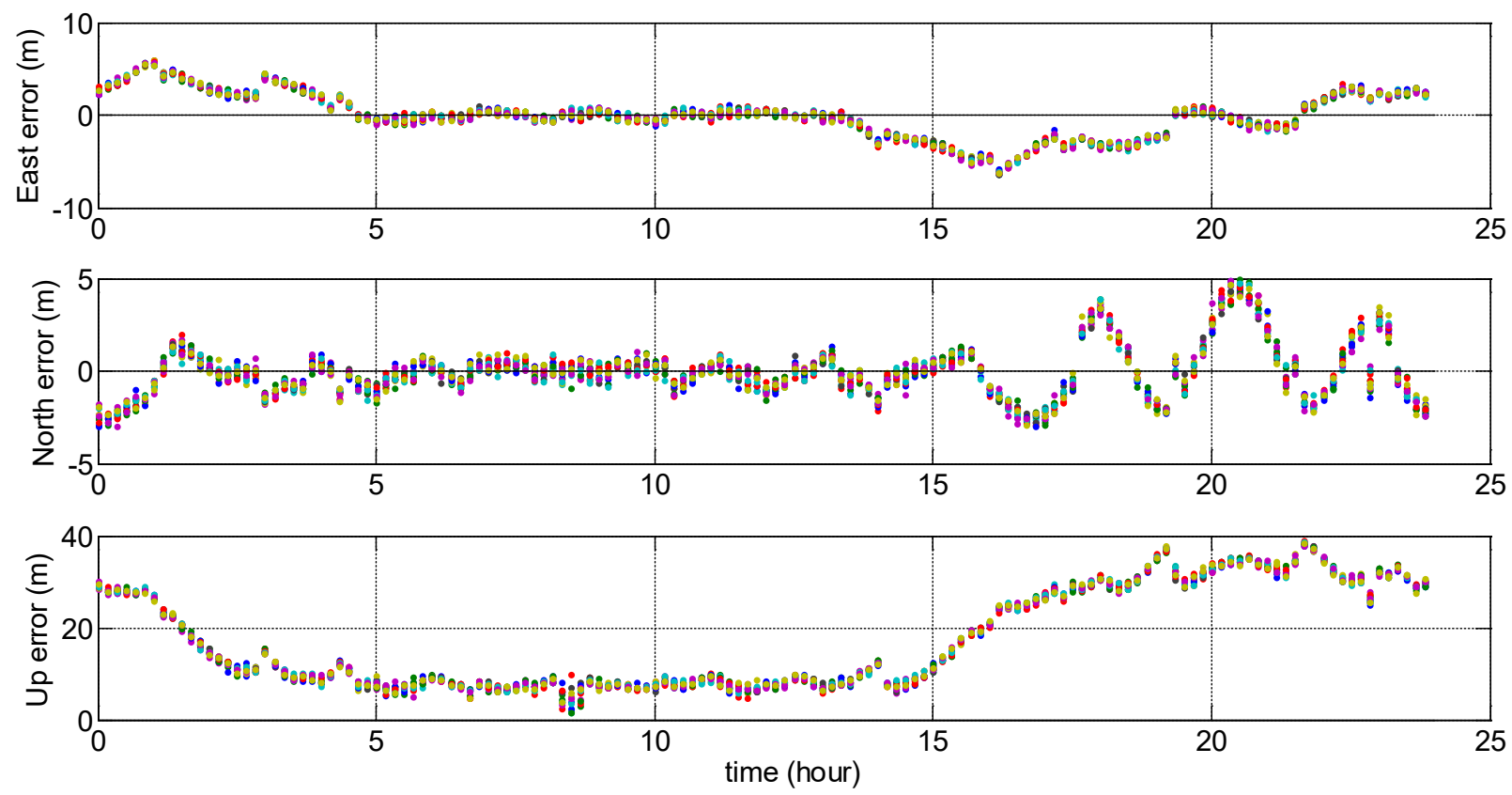

Figure 4 Distribution of 3D GNSS errors for each receiver as a function of time. Most of GNSS error is space-correlated i.e., similar for any receiver operating in the worksite.
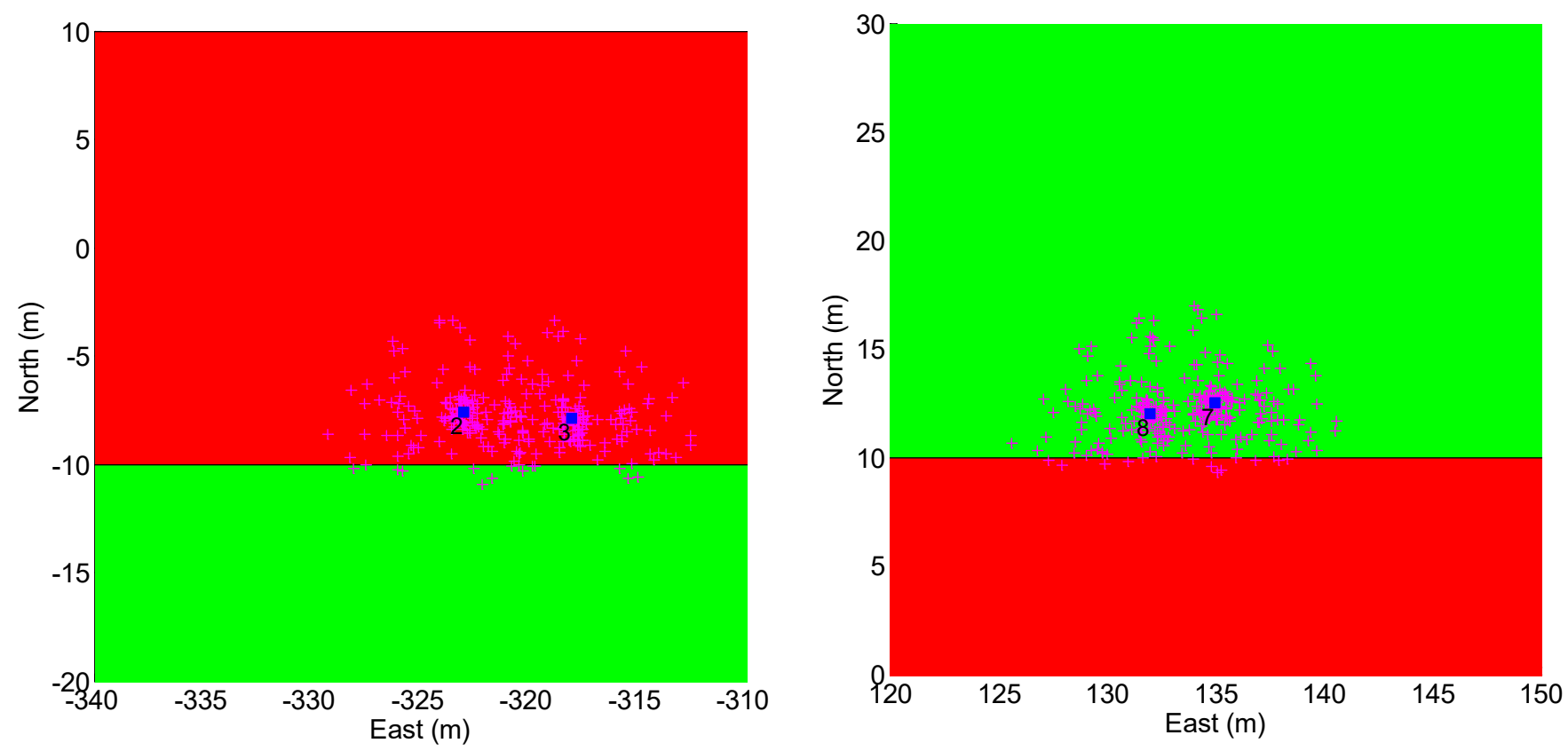

Figure 5. Example of GNSS positioning accuracy. Magenta "+" markers shows GNSS-only positions estimated in the simulated one-day long time period. Depending on actual pseudorange errors, worker located in close proximity to green-to-red zone boundary can generate both false negative (left) and false positives (right). 


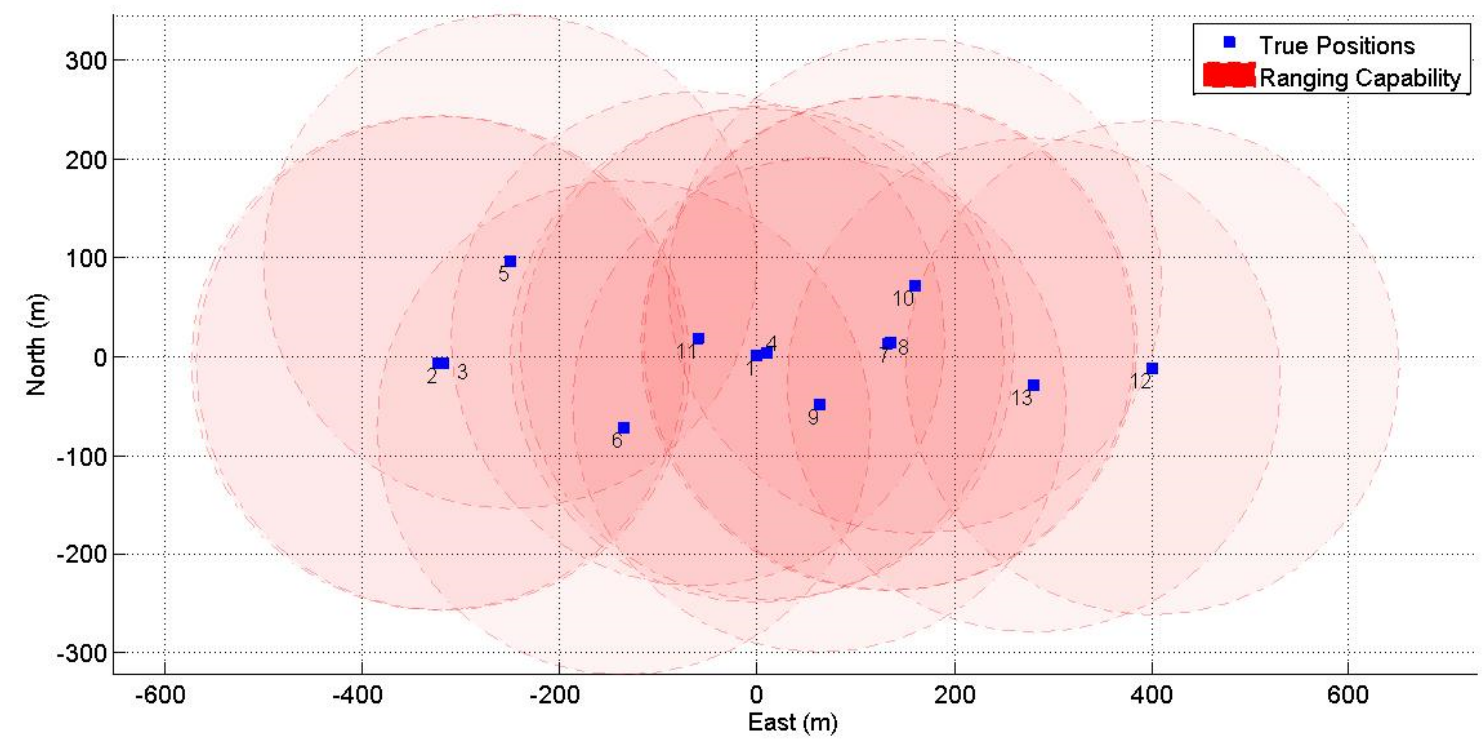

Figure 6 Relative positioning scenario assuming ranging sensors working with $250 \mathrm{~m}$ maximum range. Red circles centered in the true workers' locations represent, for each device, the area from which ranging measurements can be collected.

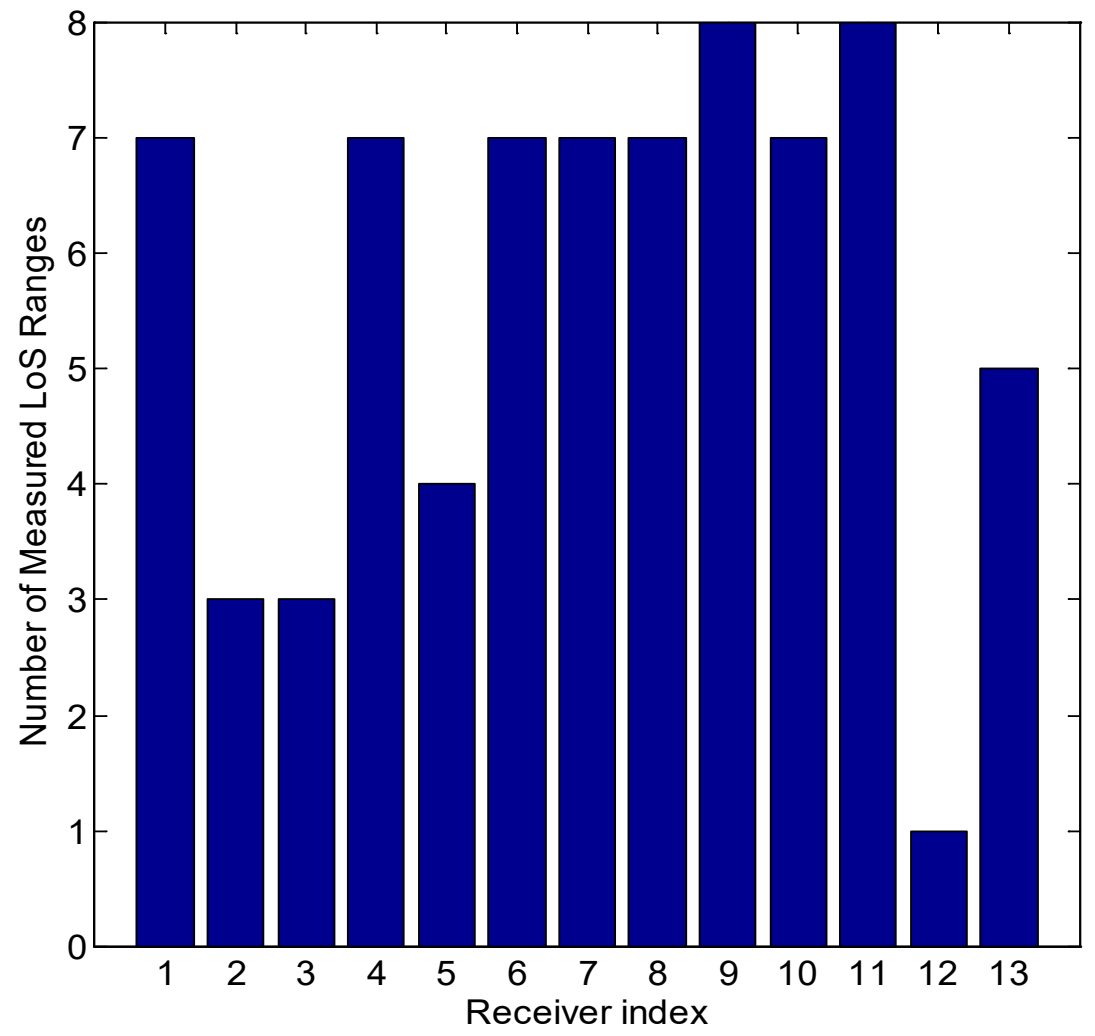

Figure 7 Number of available ranging measurements (ranging sensors working with $250 \mathrm{~m}$ maximum range and workers distribution as in Figure 6).

Figure 8 shows error dispersion considering either GNSS-only, or data fusion of GNSS and ranging measurements. Specifically, the same workers as in Figures 5-6 are illustrated, and $1 \mathrm{~m}(1 \sigma)$ error is assumed for the ranging device. It is evident that data fusion is actually able to reduce error dispersion. Such a reduction is quantified in Figure 9 where the RMS value of the distances 
from the true to the estimated positions is shown for all the simulated workers. Augmented GNSS outperforms GNSS-only for all the receivers. The highest performance improvement is obtained for receiver 7, for which the RMS values drops from $2.7 \mathrm{~m}$ with GNSS only to $0.5 \mathrm{~m}$ with augmented GNSS. Similar results hold for receivers 1,4 , and 8 , too. The variation of augmented GNSS performance among different receivers can be explained arguing that the improvement guaranteed by ranging devices depends on two main factors: (i) number of available ranging measurements and (ii) relative geometry of the available ranging measurement with respect to the current receiver. Comparing Figure 7 and Figure 9, the effect of the number of receivers is clear. For instance, receiver 12, which has got just 1 ranging measurement available, cannot achieve a significant reduction of RMS value. The effect of relative geometry can be instead observed comparing Figures 6-7 and Figure 9. With specific reference to receiver 10, even if the receiver can use the same number of ranging measurements as receiver 7, the obtained RMS value is $2 \mathrm{~m}$, i.e. $1.5 \mathrm{~m}$ worse. This is because all the available ranging measurements are relevant to receivers that are placed at lower north coordinates. Such a distribution does not allow ranging augmentation to correct GNSS only errors affecting the north component, thus resulting in large north errors, which in turn affect the RMS value.

Based on this analysis, it is possible to state that GNSS only performance is significantly improved by ranging augmentation when any given receiver can use ranging measurements coming from different directions in the horizontal plane. The result suggests that using additional receivers and ranging systems, i.e. acting as pseudo-workers, regularly displaced along the edges of the working site, enable accurate positioning of the all the receivers, i.e. true-workers, operating inside the site.
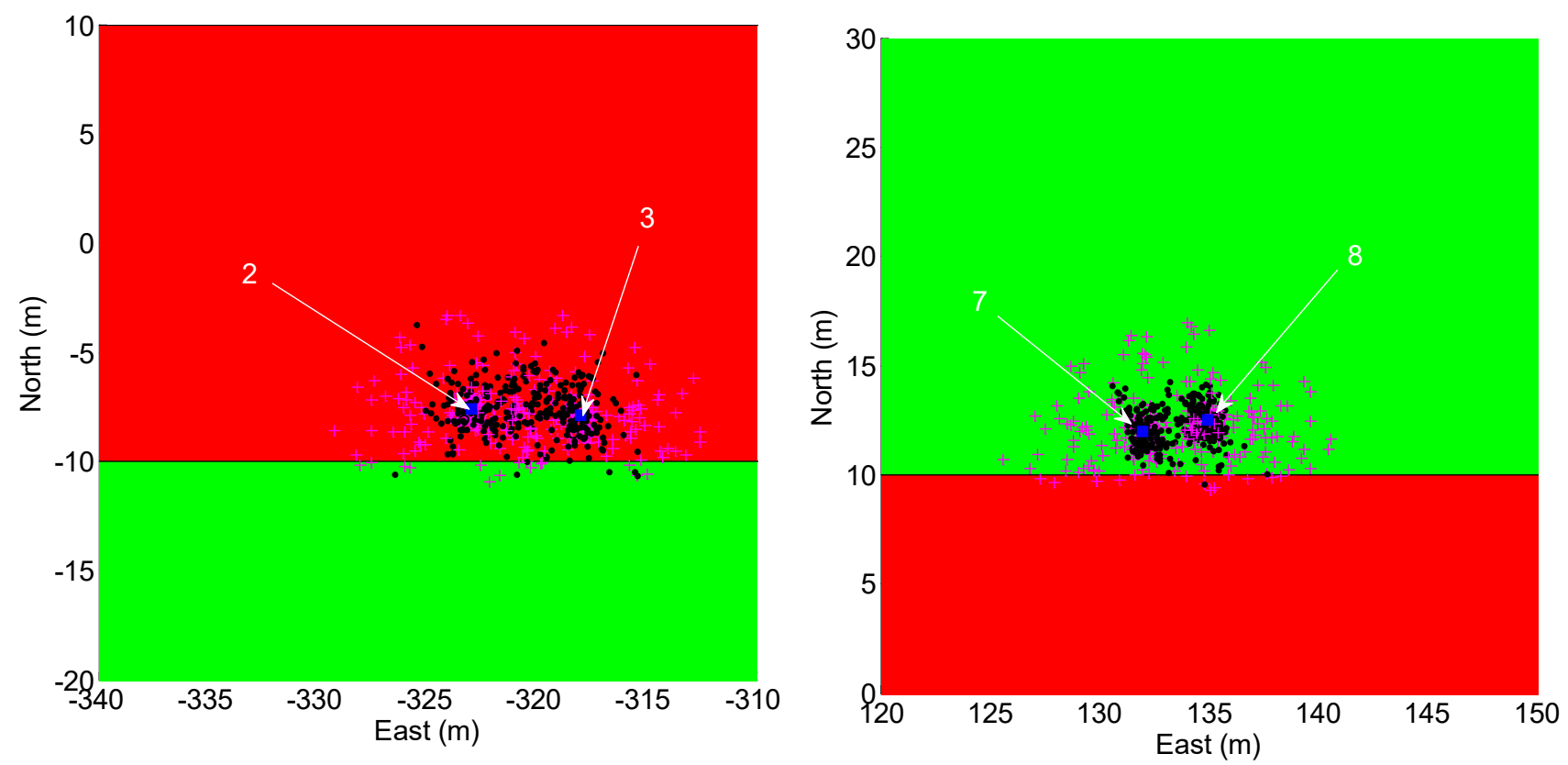

Figure 8 Comparison between GNSS-only and fusion of GNSS and ranging measurements. The cases of workers prone to generate both false negative (left) and false positives (right) are depicted. Magenta "+" markers shows GNSS-only positions estimated in the simulated one-day long time period, whereas black dots represent the output of the proposed data fusion algorithm. Error dispersion is reduced by data fusion. 


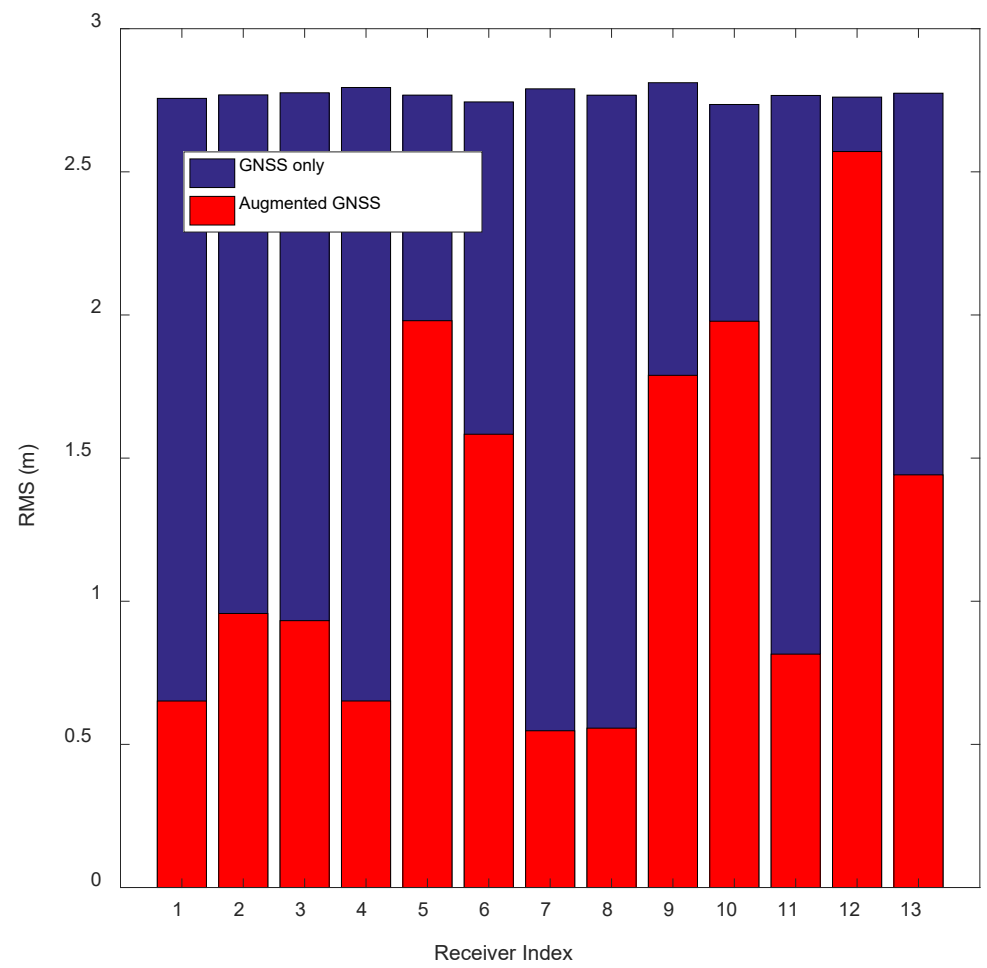

Figure 9 RMS values of the distances from the true to the estimated positions for both GNSS only (blu) and Augmented GNSS (red). Ranging sensors working with $250 \mathrm{~m}$ maximum range and $1 \mathrm{~m}(1 \sigma)$ error. Workers distribution as in Figure 6.

In order to compare the performance of different candidate ranging systems, a parametric analysis is conducted, simulating different values of maximum range and measurement error for the ranging device. Results are resumed in Figure 10. Significant reduction of the number of false positives is achieved with respect to GNSS-only operation. Specifically, the false positive rate is reduced from $40 \%$ to $95 \%$ with the increasing maximum range and ranging accuracy. Finally, the number of false negative decreases from 15 to 2 i.e., more than $85 \%$ reduction, when high accuracy ranging sensors can operate with more than $250 \mathrm{~m}$ maximum range.

From the perspective of building solutions for workers' safety, these results represent a significant step forward, especially for what concern the reduction in false negative which is the most important concern.

However, it could be argued that some false negative may still occur, leading to a hazard rate that is still considered high in safety-critical systems. This problem can be easily mitigated introducing a buffer zone at the borders of the red-to-green zones: a worker located in the buffer zone is automatically marked as in red zone. Although this will increase false positives, it will allow drastically reducing false negatives. 

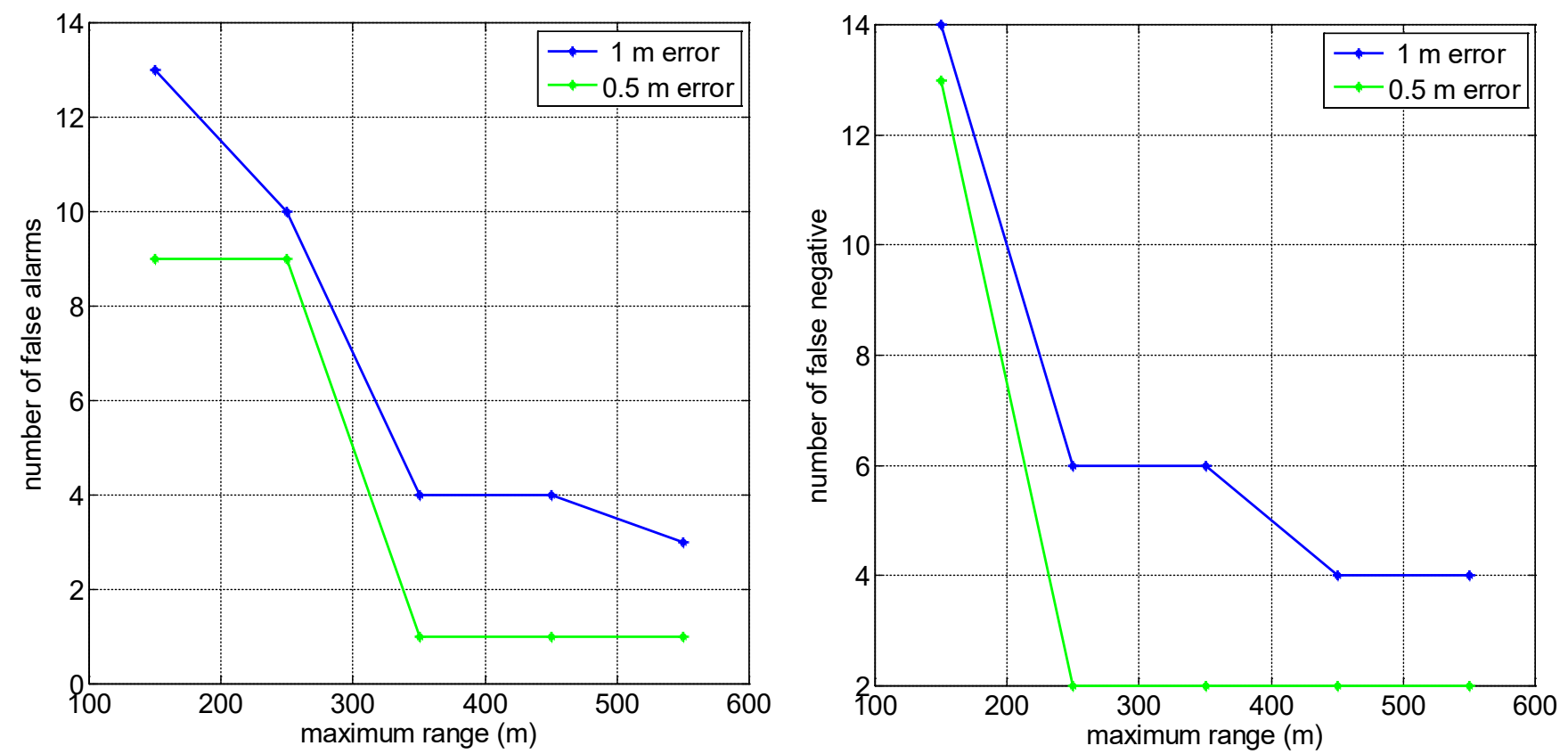

Figure 10 Number of false positives (left) and false negatives (right) as a function of maximum range and error of the selected ranging system. Worksite and worker's distribution is that shown in Figure 1.

\section{APPliCATION TO THE RAILWAy DOMAIN AND COMPLIANCE WITH STANDARDS}

\section{A. Railway domain}

A railway worksite is required for trackside maintenance workers to operate on the tracks. Worksites are set up and dismantled rapidly, and they are expected to be easily movable to rapidly shift to adjacent segments of the track. Railway worksites may be in isolated, remote places, where bringing, placing, and moving equipment (e.g., antennas relays, radars, electronic fences, etc.) is exceedingly time-consuming. As in the simulated scenarios, the worksite is divided in a safe zone, also called green zone, and in a dangerous zone, or red zone, that typically comprises the tracks and the nearby area. The size of the red zone varies according to National regulations. For example, the safe working limit is $2.0 \mathrm{~m}$ by the inner side of the rail in UK [18], $1.9 \mathrm{~m}$ by the center of the track in Germany [19], and $1.5 \mathrm{~m}$ by the inner side of the rail in Belgium [20]. When a train is approaching the worksite, all workers are alerted to move to the green zone. This alerting time is usually of few seconds: for example, it is a minimum of 10 seconds in UK [18], 15 seconds in Belgium [20] and 35 seconds in Germany [19].

Traditionally, notifying a train approaching the worksite is a task assigned to human lookouts [21]. Alternatively, solutions as Automatic Track Warning Systems (ATWSs, [17]) can be exploited. When building an ATWS, it is evident that accurately knowing the location of the workers would help to raise the worker attention and improve its safety: for trackside maintenance workers, being struck by a train or a rolling stock can be fatal and only requires a momentary lapse in attention [17].Being able to accurately localize the worker in the red or green zone would have a significant impact on ATWS functionalities and on the consequent worksite safety. It should be remarked that any proposal of localization function for safety-critical devices must be compliant to the prescriptive safety standards of the railway domain. 


\section{B. Compliance with safety standard}

The GNSS augmentation approach is analyzed in the light of the requirements of the safety standards for electronic equipment and communication in the railway domain, namely the standards EN 50126 [23] for the lifecycle of safety-critical systems, EN 50128 [24] for safety-critical software, EN 50129 [25] for safety-critical hardware, and EN 50159 [26] for safe communications.

To this end it is worth highlighting that the methodology and the algorithm taken into account can be implemented through software or even optimized in hardware, and validated following the lifecycle recommended by the standard EN 50126 [23], without expecting relevant deviations. As general safety considerations, it can be remarked that the probability of detecting a worker in the green zone while instead he is in the red zone (false negative) should be minimized, to an extent that depends on the Safety Integrity Level (SIL). The latter is a number of defined discrete levels for specifying the safety integrity requirements of the safety functions, to be allocated to the safety related systems [23] of the ATWS.. Instead, false positives, i.e. workers detected in a red zone while they are in the green zone, may cause work interruption, but they do not originate safety issues and consequently they are acceptable from a safety perspective. Also, the proposed approach is able to locate the worker minimizing the number of false negatives, which can be further reduced by introducing a buffer zone at the borders of the red-to-green zones, although at the cost of a higher number of false positives.

Moreover, to discuss compliance with the standard it is necessary to have some credible discussion on possible architectural choices.

For the wearable sensors that constitute the proposed solution, a safe failure should be implemented by signaling the worker to move to a green zone. Differently from most safety-critical systems, the realization of the safe failure is not a shutdown of the wearable sensor, but it is the activation of a notification mechanism. How to build such notification mechanism is outside the scope of this paper, but a possible approach can be found in [6]. In brief the approach is based on actuators realized by redundant notification channels that exploit different senses of the worker, such as eyesight and hearing. Alternative solutions, although not applied to the railway domain, are in [27], [28].

The software, designed, developed and validated in according to the standard EN 50128 [24], has to follow the appropriate coding rules as the MISRA-C guidelines for the use of C language in safety critical systems [29], and it has to be tolerant to residual software faults. Apart from additional engineering effort, the application of the requirements of EN 50128 does not impact on the realization of the proposed approach.

Concerning hardware, instead, the standard EN 50129 [25] proposes composite, reactive or inherent fail safety mechanics; their selection is dependent on the architecture of the wearable sensor and on the selected SIL [23].

Relevant considerations are instead devoted to the introduction of off-the-shelves (OTS) software and hardware, and in particular the GPS sensor and the communication antenna, with the related software drivers. In fact, for cost constraints, it is generally preferable to reuse existing solutions rather than built new ones from scratches. It is required to timely detect any malfunction of such sensors and prevent that the malfunction propagates through the system. In the practice, this can be realized through several mechanisms, from credibility checks to components wrapping. Further, choosing well-known OTS sensors that have been largely used in the past and are produced by credible vendors, offers the possibility to produce certification evidence using vendor's data and proven-in-use statements.

Finally, the safe communication between the different nodes also requires attentive considerations. The risk of unauthorized access to the transmission system is not negligible: consequently, our system shall operate as an open system according to the standard EN 50159 [26]. The introduction of a safety layer is necessary, to protect integrity, authenticity, timeliness and sequencing of the transmitted data following the prescriptions of EN 50159 [26]. These properties are easily achievable with state-of-the-art solutions, considering that the number of pieces of connectable equipment, and the characteristics of the transmission system, are 
known and fixed. Finally, while confidentiality and reliability of the communication would be desirable, their absence is not a safety issue [26]. For example, from a safety perspective, denial of service attacks, as wireless jamming, are reduced to a failure of the wearable sensor, and should lead to a safe failure, with the effects previously described.

\section{CONCLUDING REMARKS}

Accurately localize railroad workers allows deciding if they are in a dangerous area or not or, in other words, if they are at risk. Such localization feature would significantly boost the state of the art on automatic track warning systems, which generally do not rely on workers position due to the difficulties in accurately localize workers in railway worksites. In fact, limiting factors are that GNSS alone is not sufficient, and anchor-based approaches are difficult to exploit due to costs, battery efficiency, or set-up and dismantle time.

The augmented GNSS system based on low-power consumption wearables sensors and data fusion exploits cooperation between workers to infer if they are in a critical area. Simulation results show that the proposed solution is capable of identifying workers in safe and unsafe zones reducing the number of false positives at least of $40 \%$ and sometimes even of $95 \%$ with respect to systems exclusively based on the GNSS technology.

Although the proposed solution is specifically intended for railway workers safety, its application can be envisioned also in other domains, where i) the requirements for personnel safety are similar, and ii) usefulness of acquiring the position of personnel is acknowledged.

\section{ACKNOWLEDGEMENTS}

This work was supported in part by the POR-CREO REMOTE project, and by the FP7-IRSES-DEVASSES project.

\section{REFERENCES}

[1] Mao, G., Fidan, B., and Anderson, B. D. 2007. Wireless sensor network localization techniques. Comput. Netw. 51, 10 (Jul. 2007$), 2529-2553$.

[2] Occupational Safety and Health Administration, “'Struck-by’ hazards - Trainer guide”, 2011, https://www.osha.gov/dte/grant_materials/fy07/sh-1658607/2_struckby_hazards_trainer_guide.pdf.

[3] E. D. Kaplan and C. J. Hegarty (Eds.), "Understanding GPS: Principles and Applications," Second Edition, Artech House, Boston, MA, 2006.

[4] Bondavalli, Andrea, et al. "Experimental assessment of low-cost GPS-based localization in railway worksite-like scenarios." Measurement 46.1 (2013): 456466.

[5] W. R. Richards, K. O’Brien, D. C. Miller, New Air Traffic Surveillance Technology, Boeing Aeromagazine, Vol 2, 2010, pp. 6-13, [Online] http://www.boeing.com/commercial/aeromagazine/articles/qtr_02_10/2/

[6] Ceccarelli, Andrea, et al. "Design and implementation of real-time wearable devices for a safety-critical track warning system." High-Assurance Systems Engineering (HASE), 2012 IEEE 14th International Symposium on. IEEE, 2012.

[7] Figueiras, Joao, et al. "GPS and electronic fence data fusion for positioning within railway worksite scenarios." High-Assurance Systems Engineering (HASE), 2012 IEEE 14th International Symposium on. IEEE, 2012.

[8] S. Torres-Guijarro, E. Vázquez-Fernández, M. Seoane-Seoane, J.A. Mondaray-Zafrilla "A traffic radar verification system based on GPS-Doppler technology" Measurement, 43 (10) (2010), pp. 1355-1362.

[9] Ting.-Hua. Yia, Hong.-Nan. Lia, Ming. Gub "Effect of different construction materials on propagation of GPS monitoring signals" Measurement, 45 (5) (2012), pp. 1126-1139. 
[10] M.R. Kaloop, Hui Li “Sensitivity and analysis GPS signals based bridge damage using GPS observations and wavelet transform” Measurement, 45 (5) (2012), pp. 927-937.

[11] Farrell, J. (2008). Aided navigation: GNSS with high rate sensors, McGraw-Hill, New York.

[12] M. Kayton, W. R. Fried, Avionics Navigation Systems, Second Edition, John Wiley \& Sons, Inc, 1997.

[13] GPS SPS Performance Standard (4th edition, September 2008)

[14] GPS World Receiver Survey, [Online] available at: http://gpsworld.com/resources/gps-world-receiver-survey/ (latest access Novemeber, 2017)

[15] GPSoft, SATNAV Toolbox 3.0, http://gpsoftnav.com/products/satellite-navigation-satnav-toolbox-3-0/ (online)

[16] GPS Navstar Joint Program Office, Navstar GPS Space Segment/Navigation User Interfaces, IS-GPS-200, Revision D, El Segundo, CA, December 7, 2004.

[17] Noorudheen, Nusra, et al. "Keeping track workers safe: a socio-technical analysis of emerging systems and technology." Proceedings of the Institution of Mechanical Engineers, Part F: Journal of Rail and Rapid Transit 227.5 (2013): 517-528.

[18] Office of Rail Regulation (2005) Annual report on railway safety. http://www.rail-reg.gov.uk/upload/pdf/296.pdf. Accessed 22 March 2012

[19] GUV-R 2150 (1998) Sicherungsmaßnahmen bei Arbeiten im Gleisbereich von Eisenbahnen. Deutsche Gesetzliche Unfallversicherung (DGUV)

[20] Fascicule 63-Mesures de securitée et de sante lors de l'execution de travaux geres par Infrabel. Infrabel

[21] CRC for railway innovation, Track Worker Protection Technology, 2014.

[22] Ceccarelli, Andrea, et al. "Design and implementation of real-time wearable devices for a safety-critical track warning system." High-Assurance Systems Engineering (HASE), 2012 IEEE 14th International Symposium on. IEEE, 2012.

[23] CEI EN 50126 - Railway applications - The specification and demonstration of Reliability, Availability, Maintainability and Safety (RAMS), 2000.

[24] CEI EN 50128 - Railway applications - Communication, signalling and processing systems - Software for railway control and protection systems, 2011.

[25] CEI EN 50129 - Railway applications - Communication, signalling and processing systems - Safety related electronic systems for signalling, 2004.

[26] CEI EN 50159 - Railway applications - Communication, signalling and processing systems - Safety related communication in transmission systems, 2010.

[27] Corbellini, Simone, Franco Ferraris, and Marco Parvis. "A system for monitoring workers' safety in an unhealthy environment by means of wearable sensors." Instrumentation and Measurement Technology Conference Proceedings, 2008. IMTC 2008. IEEE. IEEE, 2008

[28] Senyurek, Levent, et al. "Monitoring workers through wearable transceivers for improving work safety." Intelligent Signal Processing (WISP), 2011 IEEE 7th International Symposium on. IEEE, 2011.

[29] MIRA Limited, MISRA C 2012 - Guidelines for the use of C language in critical systems, March 2013. 